Supporting Information

\title{
Seed-Mediated Synthesis of Pd Nanocrystals: The Effect of Surface Capping on the Heterogeneous Nucleation and Growth
}

Hsin-Chieh Peng, ${ }^{\dagger}$ Zhuoming Li, ${ }^{\star}$ Gregory Aldahondo ${ }^{\dagger}$ Hongwen Huang, ${ }^{\S}$ and Younan Xia ${ }^{\dagger, \star,},{ }^{*}$

${ }^{\dagger}$ School of Chemistry and Biochemistry, Georgia Institute of Technology, Atlanta, Georgia

30332, United States

\$School of Chemical and Biomolecular Engineering, Georgia Institute of Technology, Atlanta,

Georgia 30332, United States

${ }^{\S}$ The Wallace H. Coulter Department of Biomedical Engineering, Georgia Institute of

Technology and Emory University, Atlanta, Georgia 30332, United States

*Corresponding author: younan.xia@bme.gatech.edu. Phone:404-385-3209 

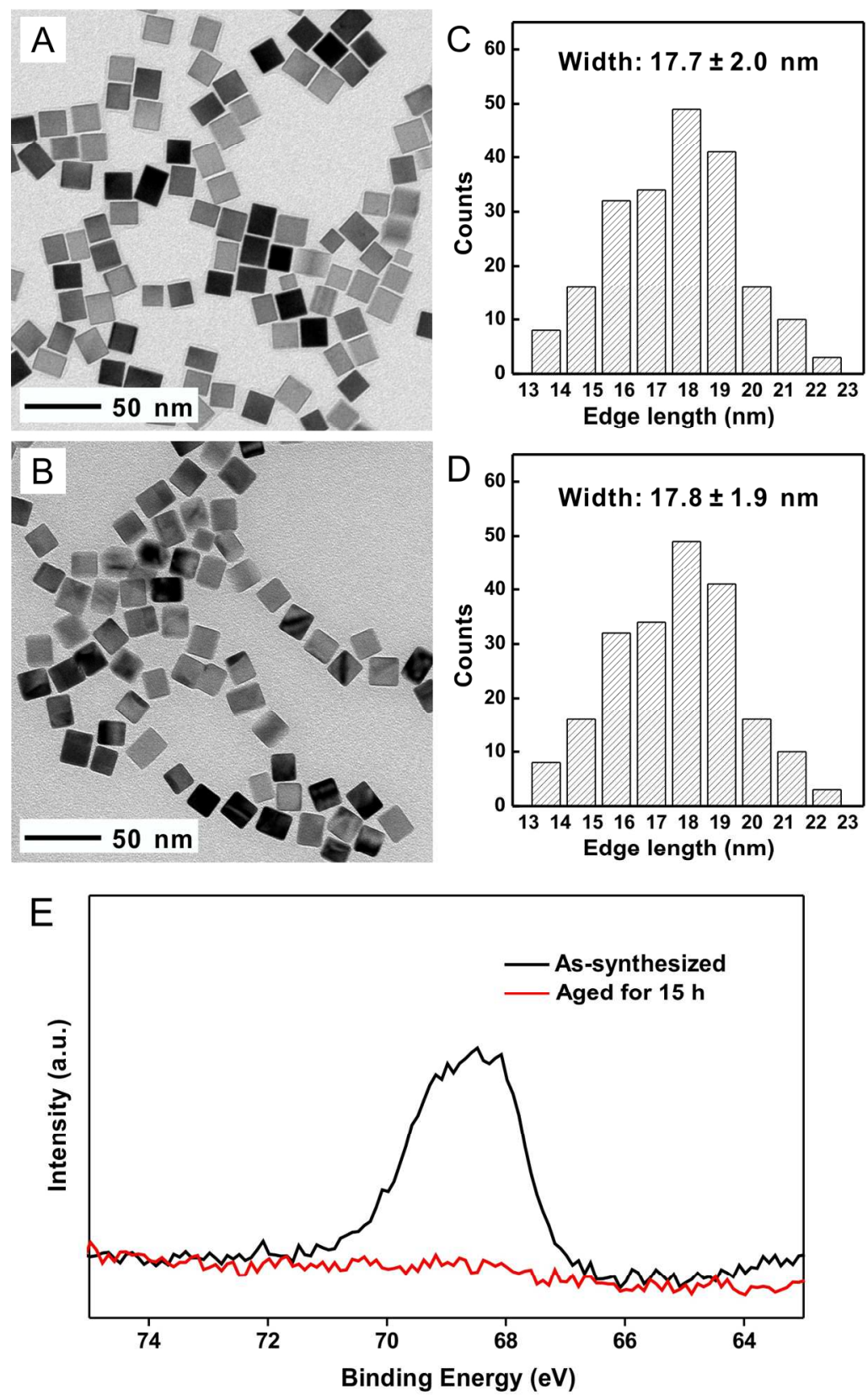

Figure S1. TEM images of 18-nm Pd cubic seeds (A) before and (B) after being aged in ethylene glycol at $120^{\circ} \mathrm{C}$ for $15 \mathrm{~h}$ to remove the $\mathrm{Br}^{-}$ions chemisorbed on the surface during the synthesis. (C, D) The corresponding statistical analysis of size distribution for each sample. (E) Br 3d XPS spectra of the Pd cubic seeds before and after the removal of chemisorbed $\mathrm{Br}^{-}$ions. 


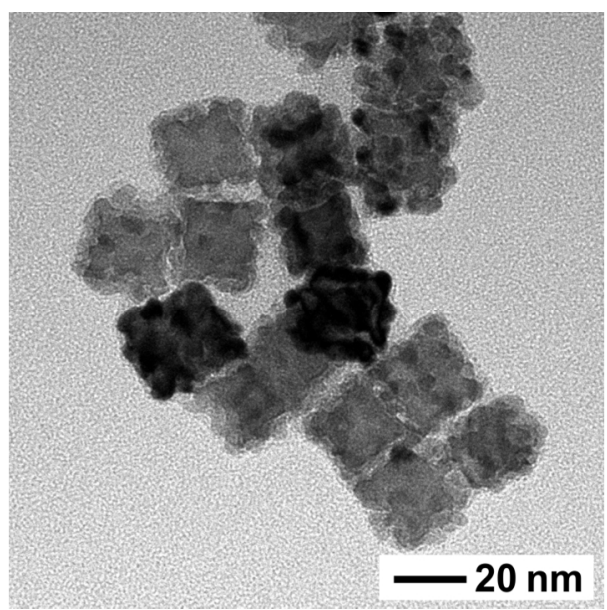

Figure S2. TEM image of Pd nanocrystals prepared under the same condition as in Figure 2C, except that the sample had been aged in the reaction solution at room temperature for another 90 $\min$. 

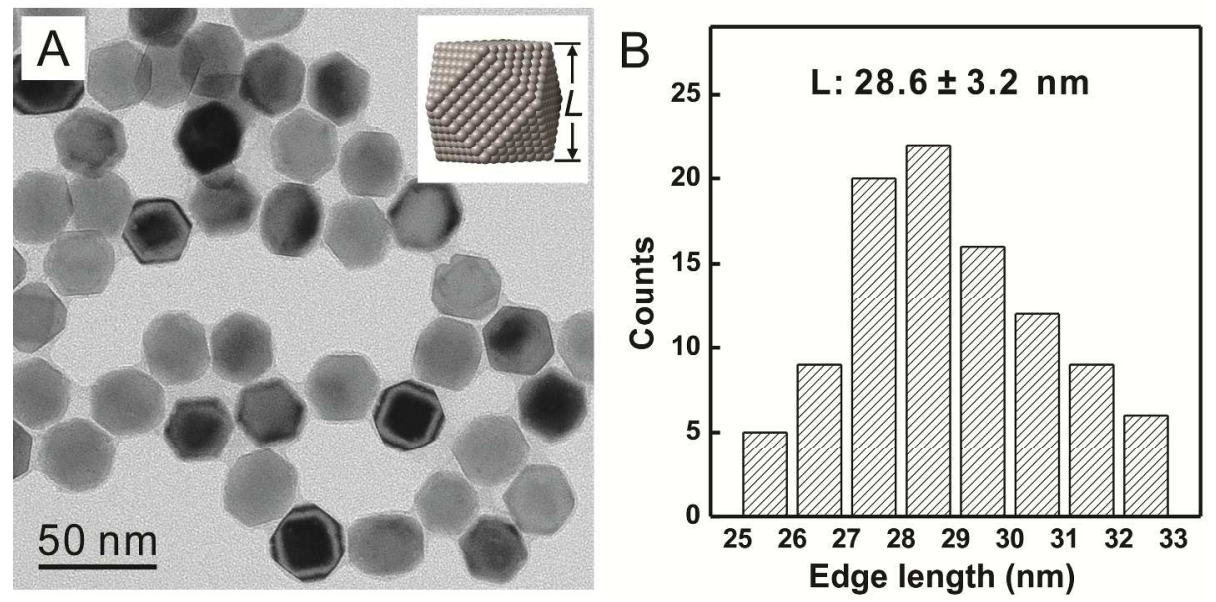

Figure S3. (A) TEM image of the as-synthesized cuboctahedral seeds with an average size (L) of $28.6 \mathrm{~nm}$ and (B) the corresponding statistical analysis of size distribution of the sample. The inset in (A) shows an atomic model of a cuboctahedron and the definition of size. 

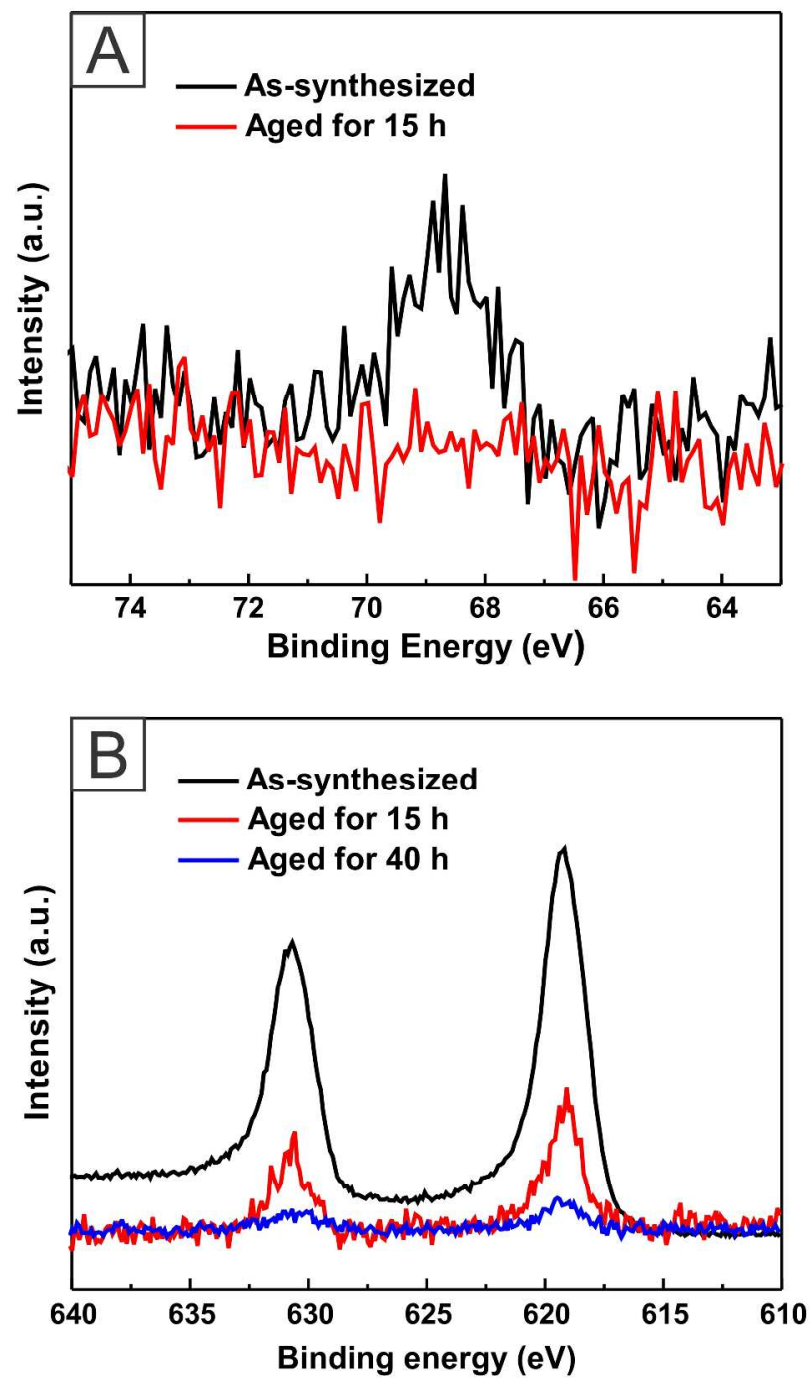

Figure S4. (A) $\mathrm{Br} 3 \mathrm{~d}$ XPS spectra of the Pd cuboctahedral seeds before and after aging in ethylene glycol at $120{ }^{\circ} \mathrm{C}$ to remove the chemisorbed $\mathrm{Br}^{-}$ions. (B) I 3d XPS spectra of the Pd nanowire seeds before and after aging in ethylene glycol at $160{ }^{\circ} \mathrm{C}$ for two different intervals of time. 